\title{
The Trend of COVID-19 at Bengaluru: Prediction to Continue the Better Epidemic Management
}

\section{Kannamani Ramasamy*1, S. Jayakumar ${ }^{2}$}

\section{ABSTRACT}

Aim: The primary objective of this study is to understand the current situation of COVID-19 at Bangalore city and predict the future state, which will help for better management.

Methods: Data from BBMP and Karnataka's government from 1 April to 12 June 2020 used to calculate the prediction. We have used FORECAST.ETS function in Microsoft EXCEL to predict the future number of COVID-19 cases for Bangalore city.

Results: Based on the prediction analysis, the number of cases at Bangalore may reach 3240 by the end of Aug 2020 . The percentage of positive cases at Bangalore from the total samples tested across Karnataka may reach up to $0.6 \%$. Death count due to COID-19 may touch at 80 . Overall, the prediction appears that the status of the COVID-19 may continue to be good with some additions.

Conclusion: Bangalore is doing well with COVID-19 management. However, the situation may change at the point of time due to various contributors. Proactive approaches, as mentioned in the recommendations section, is much critical to managing the pandemic situation.

Key Words: Bangalore, COVID-19, Coronavirus, BBMP, Trend, Prediction

\section{INTRODUCTION}

Coronaviruses are a large family of viruses which may cause sickness in animals or humans. Several Coronaviruses are identified in humans to cause respiratory infections ranging from the typical cold to severe diseases. This new virus COVID-19 and disease were identified in Wuhan, China, in December 2019. The common indications of COVID-19 are fever, dry cough and tiredness. Some patients may have aches and pains, sore throat nasal congestion, sore throat or diarrhoea and runny nose. These symptoms are usually mild and improve gradually. Some people become infected but don't show any signs and they appear to be well. Aged people and those going through medical problems like high blood pressure, cardiovascular issues or diabetes, are more likely to grow serious illnesses. People who have the indications of dry cough, fever, and difficulty in breathing must consult with the medical professional ${ }^{1}$. Globally, India is in 4th place with 333,008 cumulative positive cases as on 15 June 2020, next to America $(2,162,228)$, Brazil $(867,882)$ and Russia $(528,964)^{2}$. In India, Karnataka is in 10th place with the 7213 (as of 15 June 2020) confirmed cases ${ }^{3}$. Within
Karnataka state, Bangalore is in 4th place with 725 cases (as of 15 June 2020) next to Udupi, Kalaburagi and Yadagiri districts ${ }^{4}$.

\section{Bangalore city - Overview}

Bangalore city is one of the fastest emerging cities in India and capital for Karnataka state. The Bangalore city is the fifth-largest city across the country. Bangalore city called 'Garden city 'of India due to the huge number of trees and plentiful greenery. Bengaluru city is also called as "Silicon Valley of India" for the development of IT and ITES sectors in India. Bangalore renamed as "Bengaluru" in 2014. Various Indian technological organizations such as Indian satellite research organizations, Infosys Limited, Wipro, and Hindustan aeronautical limited are headquartered in the city. A demographically diverse city and has highly educated workforces in the world. Bengaluru is a place where multiple educational and research institutions located such as Indian Institute of Bangalore, Indian Institute of Science, National Institute of Advanced Studies, Tata Institute of Fundamental Research, Institute for Social and Economic Change,

\section{Corresponding Author:}

Kannamani Ramasamy, Independent Researcher and Alumnus of Jain University, Bangalore, India; Mob: 9986595820; Email: Kannamani23@gmail.com

ISSN: 2231-2196 (Print)

Received: 20.06 .2020
ISSN: $0975-5241$ (Online)

Revised: 24.06 .2020
Accepted: 28.06 .2020
Published: 05.07 .2020 
International Institute of Information Technology Bangalore, National Institute of Design, R \& D Campus, National Law School of India University and The National Institute of Mental Health and Neuro-Sciences. Bangalore is located at 12.590 north latitude and 77.570 east longitude and positioned at an altitude of nine hundred and twenty meters above sea level. Bangalore city has more than 650 Indian and multinational companies. Bangalore is contributing 35\% of India's software exports 5 .

\section{Motivation}

Bangalore is the silicon valley of India. The city is well connected with all other major cities in India. On 9 March 2020, the first COVID-19 case identified at Bangalore. Bangalore is appropriately connected with the International airport with huge visitors from multiple nations and cities. As of 15 June, the city has 725 COVID-19 confirmed cases. For a population of 1.3 crores, the number of cases is pretty less $(0.005 \%)$. Though neighbouring states like Tamilnadu and Maharashtra affected severely, Bangalore can maintain the confirmed cases at a low level. This exciting pattern is making us study about the COVID-19 status at Bangalore.

\section{Present COVID-19 situation at Bangalore}

Figure 1 shows the trend of COVID-19 confirmed cases in India, Karnataka and Bangalore city, as on 13 June 2020. The first case in the state identified on 8 March 2020.

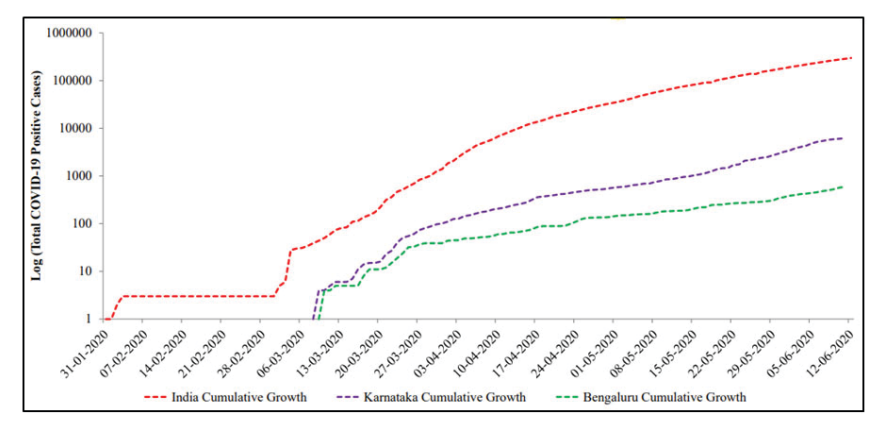

Figure 1: COVID-19 confirmed cases in India, Karnataka and Bangalore city ${ }^{3}$.

Figure 2 shows the number of COVID-19 cases for all the districts in the Karnataka as on 13 June 2020. Bangalore Urban is in $4^{\text {th }}$ place in the state next to Kalburgi, Yadagiri and Udupi districts. As of today (15 June 2020), there are 725 confirmed cases in the Bangalore city. The city is able to maintain the lower count with many initiatives ${ }^{11}$ by the authorities. Some of them are : Around 140000 people involved in international travelling were screened and they have kept under monitoring. Also, their associate contacts were kept under observation. Coordination between the civic agency, health and home departments was effective, which helped maintain the numbers at minimal. Citizens of Bengaluru are responsible, managing the social distancing and wears a mask. Bruhat Bengaluru Mahanagara Palike's mapping of containment zones and predictive modelling are vital factors for success. Bruhat Bengaluru Mahanagara Palike had taken the police department's help and protected health workers, so they were able to perform their duties ${ }^{11}$.

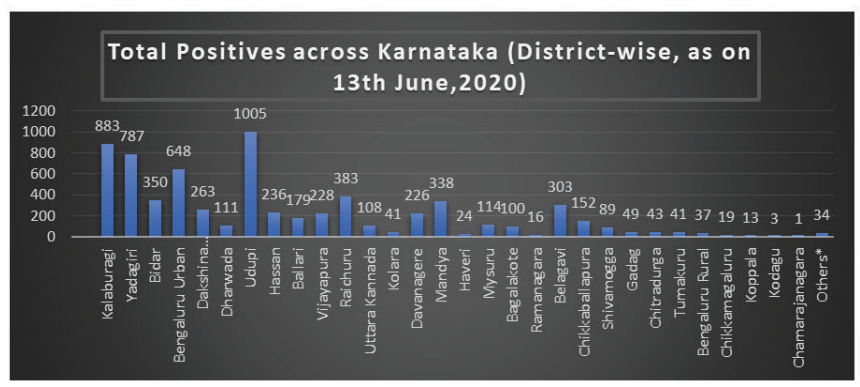

Figure 2: COVID-19 confirmed cases in across the state at Karnataka ${ }^{3}$.

Figure 3 shows the trend and the number of cases during each lockdown and also after lifting the lockdown. The first case in the state identified on 8 March. During the first lockdown (1.0), there were 32 confirmed cases in Bangalore. During the second lockdown (2.0), there were 71 cases in total. During the $3^{\text {rd }}$ lockdown (3.0), the number of cumulative confirmed cases were 153 . There were 239 cases during $4^{\text {th }}$ lockdown (4.0). During $5^{\text {th }}$ lockdown (5.0), the aggregate number of confirmed cases was 358 in Bangalore city. After lifting the lockdown, as of 15 June, the number of confirmed cases increased to 725 from 358 .

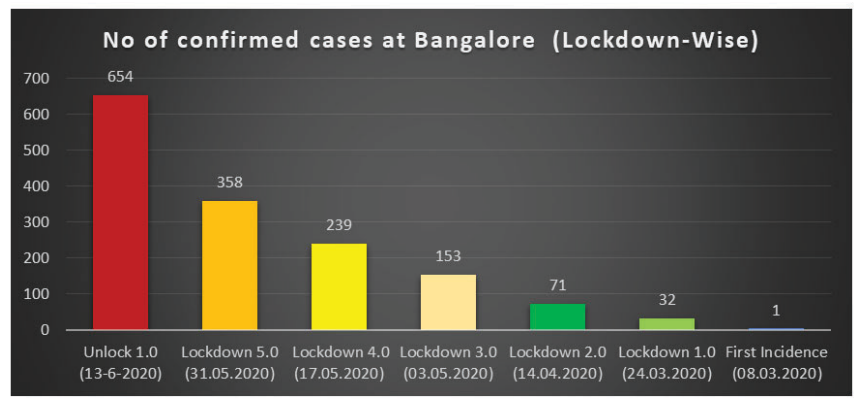

Figure 3: COVID-19 confirmed cases in Bangalore city, during and after lockdown ${ }^{3}$.

Figure 4 shows the number of confirmed cases in Bangalore from 1 April to 14 May, where we can see an increasing trend. Figure 5 shows the number of confirmed cases in Bangalore from 14 May to 13 June 2020. Figure 6 represents the trend of percentage of confirmed cases at Bangalore from the number of total samples tested on that particular day across Karnataka state. Figure 7 indicates the direction ofCOVID-19 death cases at Bangalore city from 19 April (reported 4 deaths) to 13 June 2020 (34 cumulative death cases). 


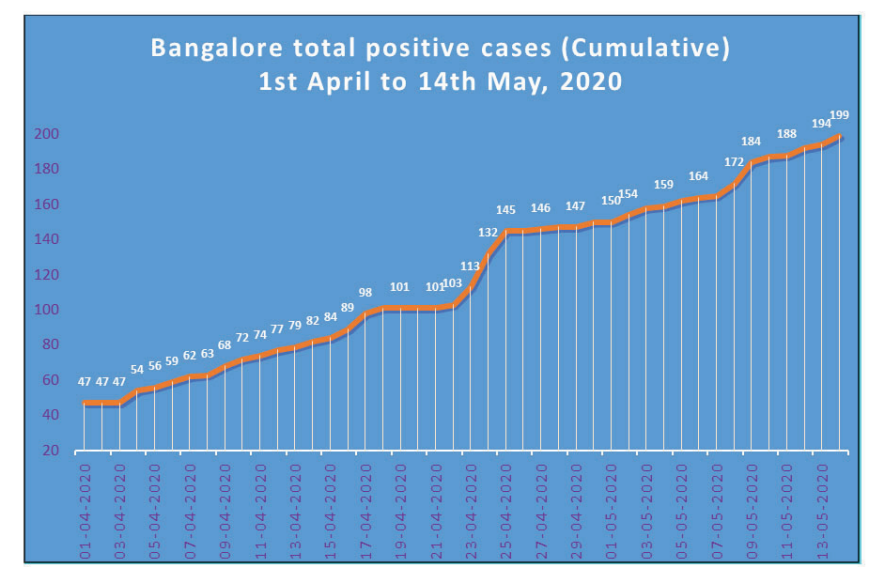

Figure 4: Trend of COVID-19 confirmed cases in Bangalore city ${ }^{4}$.

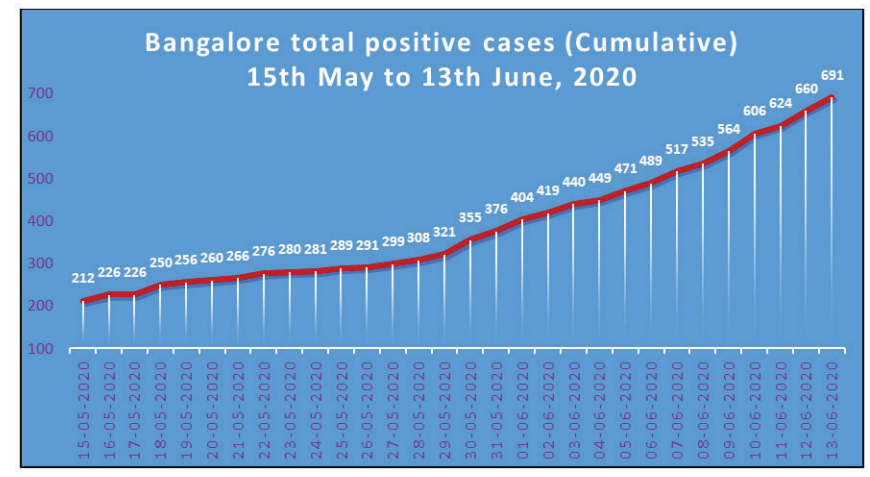

Figure 5: Trend of COVID-19 confirmed cases in Bangalore city 4 .

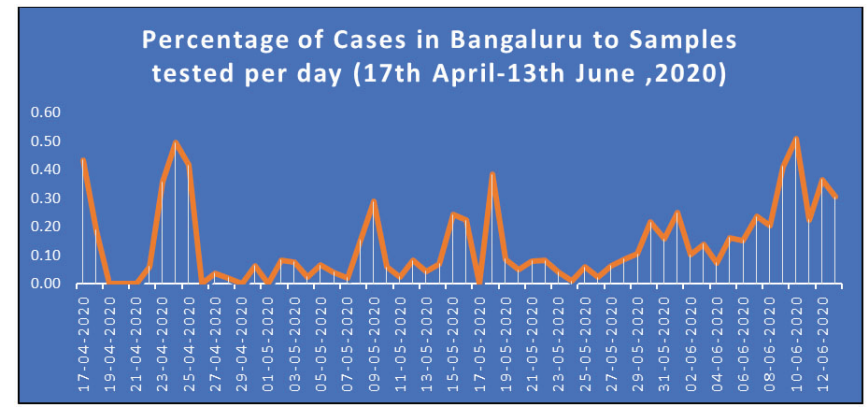

Figure 6: Trend of COVID-19 confirmed cases percentage from the sample tested ${ }^{4}$.

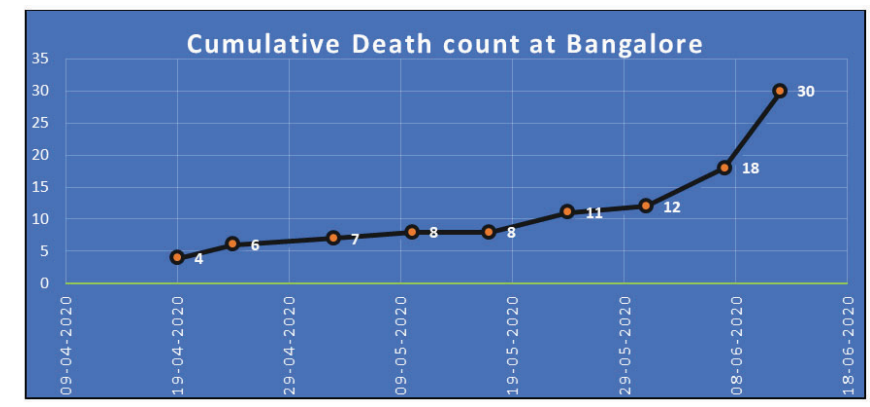

Figure 7: Trend of COVID-19 death cases at Bangalore city ${ }^{4}$.

\section{Forecasting technique used}

In this paper, we have used FORECAST.ETS function in Microsoft EXCEL to predict the future number of COVID-19 cases for Bangalore city. The Excel ${ }^{8}$ Forecast.Ets function uses an exponential smoothing algorithm to predict a future value on a timeline, based on a series of existing values. From 1 April 2020 till 13 June 2020 used as a current data. Prediction analysis calculation made from 14 June 2020 to 31 August 2020. Prediction analysis calculated for around 75 days. Calculating prediction analysis for more days (more than three months) may lead to deviation as there may be various factors that would change in the long duration. However, with 75 days duration, lower and upper bound should be able to help us to cover the variations.

\section{Prediction of COVID-19 for Bangalore}

\section{Prediction for Positive cases}

This prediction applies from 14 June to 31 August 2020. In figure 8 , bluelines are indicating the past trend and orange lines are showing the future trend. The thin lines above the orange indicate the upper limit, and the range within orange lines indicates the lower limit of the forecasting. With $95 \%$ confidence level, the prediction may vary from lower to upper numbers. Figure 9, too representing the forecasting; however, it is presented for clear visibility by using the weekly data.

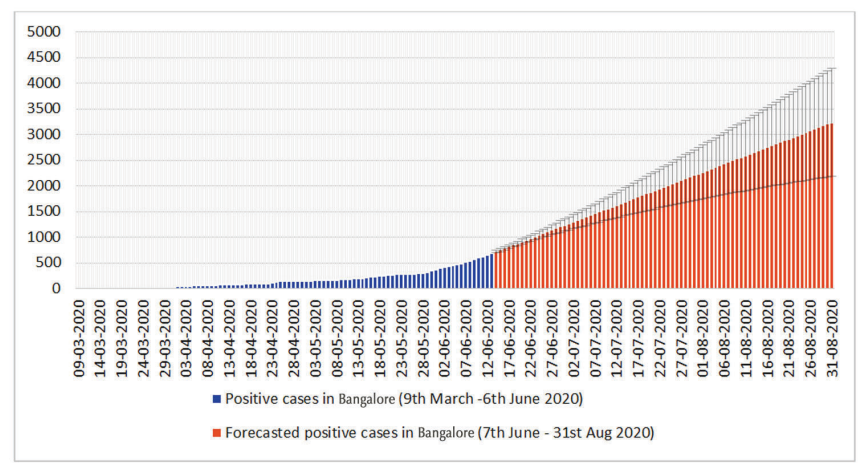

Figure 8: Forecasted COVID-19 positive cases at Bangalore ${ }^{8}$.

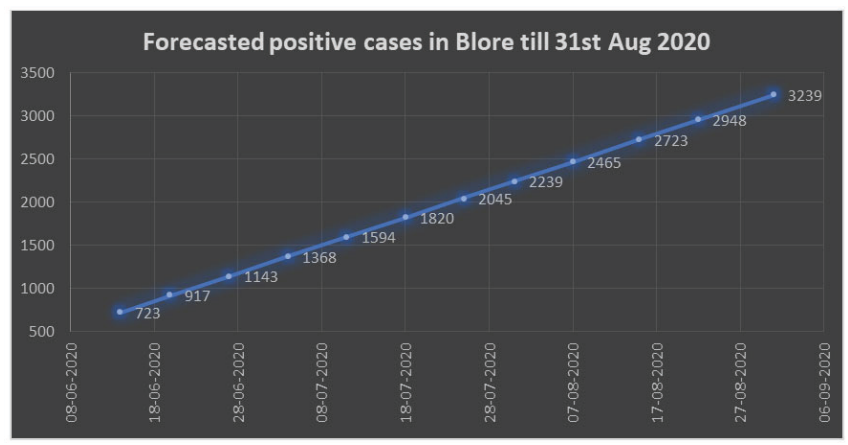

Figure 9: Forecasted COVID-19 positive cases at Bangalore ${ }^{8}$. 


\section{Prediction of Samples (positive cases) from the total test}

In figure 10, bluelines are indicating the past trend and orange lines are showing the future trend. Currently (as on 13 June 2020), it is at $0.34 \%$ and in the future (as of 31 August 2020), it may go up to $0.60 \%$.

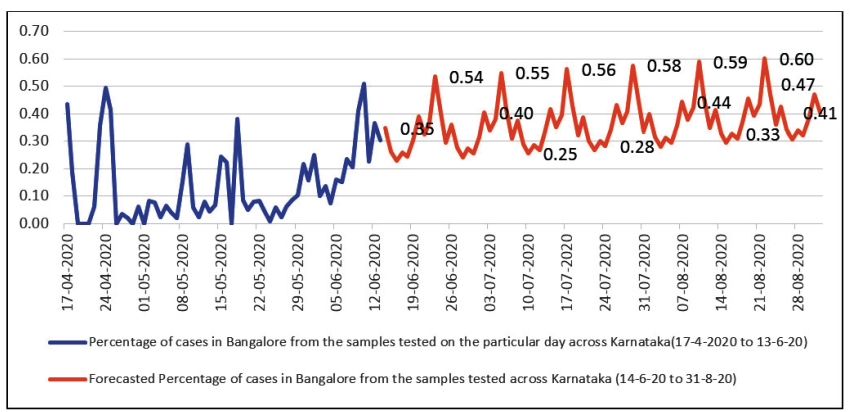

Figure 10: Forecasted COVID-19 positive samples from the total samples of $\mathrm{KN}^{8}$.

\section{Prediction of Number of Death cases}

As of 12 June 2020, the number of death cases declared by the authorities of Bangalore city is 30 . Based on the prediction analysis, the number of death count may go up to 59 by 31 August 2020 (Figure 11). In the worst scenario, it may reach up to 79 (refer upper bound in the figure, yellow colour) and with better measures, it can be controlled at 39 (refer lower bound in the figure, grey colour).

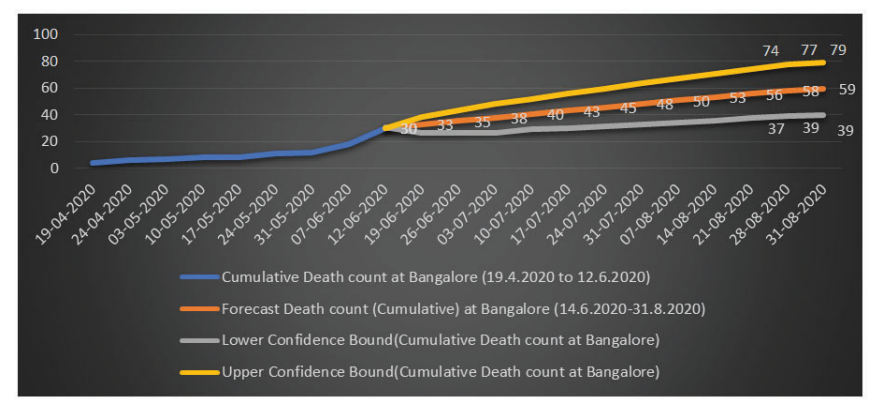

Figure 11: Forecasted COVID-19 death cases at Bangalore city ${ }^{8}$.

\section{Discussion and Interpretation}

Mumbai, which is the capital of Maharashtra, is profoundly affected by an enormous number of COVID-19 case. Chennai which is the capital of another neighbour state for Karnataka, also affected severely ${ }^{12}$. The first case of Karnataka found on 8 March 2020 and as on 15 June 2020, the number of positive cases is 7213. The number of positive cases in Bangalore is around 700 (figure 5). Though there are a massive number of IT companies, other industries and exposure to international travel, maintaining this number at Bangalore is appreciated. One side, it is essential to know that many precaution meas- ures (discussed in the section named "Present COVID-19 situation at Bangalore, reference no 11) have been taken by the government of Karnataka and BBMP at Bangalore city to maintain and control the spread. While we are happy to see the low number of positive cases, if the number of samples testing goes high, it may reveal even more positive cases. 4020 samples tested per million across Karnataka', as of 29 May 2020. Testing is the crucial element to control the spread. It is important to note that the World Health Organization sent an advisory to all countries in March to conduct more test. The more tests we do (5139 tests conducted per million at Bangalore, as of 21 June 2020), the easier it is to track the spread of the virus and reduce transmission. Many countries followed the advice, which was resulted in better outcome ${ }^{10}$. Another possible reason for low numbers of positive cases at Bangalore may be due to the educated crowd in the city. A considerable number of people are working in IT, ITES, education, and other industries where they can work from home. Hence, there are no physical contacts which helped for better social distancing.

Another side, through the cumulative confirmed cases, the situation is under control at the Bangalore city. In the past one week, the counts are kept on increasing. Till 14 May, the number of cases is 200 (figure 4) and from 15 May to 15 June, 500 cases are added within a month (figure 5). The percentage of positive cases at Bangalore from the total samples tested across Karnataka vary from 0.1 to 0.3 on average (figure 6). On 19 April, 4 death cases reported and on 12 June, the cumulative death count was 30 . (figure 7).From the lockdown 0 to 4 , the number of confirmed cases was 358 . After unlocking, the number of cumulative cases reached 700. This pattern raises another question of whether the unlocking played a massive role in increasing the confirmed cases(figure 3). We have used the FORECASTING ETS option to predict the future numbers for Bangalore city. As of 31 August 2020, the expected number of positive cases would be 3239 (figure 9) with a 95\% confidence level. Based on the conditions, the number of cases may vary, which is indicated as lower bound (between orange lines) and upper bound (Above orange lines) (figure 9). The percentage of positive cases at Bangalore from the sample tested across Karnataka may go up to 0.6 (figure 10); it may vary between 0.4 to 0.6 on an average. Currently, most of the days, it ranges from 0.1 to around 0.3 on an average (figure 10). As of $31 \mathrm{Augu}^{\text {st }}$, the number of death cases in the city would be around 59. In the worst situation, it may go up to 80 (indicated as upper bound) and it would be approximately 40 mentioned as lower bound if everything goes well and the situation is under better control. (figure 11).

\section{Recommendations:}

$\checkmark$ Current efforts by the government of Karnataka and BBMP continue to maintain the low number of positive cases concerning COVID-19. 
$\checkmark \quad$ The number of testing per million to be increased for better control and containment.

$\checkmark \quad$ Entry process and security measures to be strengthened at the borders of Bangalore city.

$\checkmark \quad$ BBMP and Government of Karnataka to advise the IT, ITES industries to continue the work from home for a few more months.

$\checkmark$ Until there is a situation that can give the officials, medical experts, and citizens confidence, authorities should not allow educational institutes to operate.

\section{CONCLUSION}

Performing well is appreciated; however, continuous performance is essential, which is not easy. Possibly with the current measures and additionally, the recommendations mentioned in the discussion and recommendations section, Bangalore can maintain the COVID-19 positive cases at a low level. But it does not mean that it will continue forever. Disaster may knock the door at any time. Preparedness for the bad situation will help to react to the situation with the proactive plans. An individual or administrator can succeed only with the proper forecasting about the future. It is advisable to calculate the forecasted numbers time to time and plan accordingly for city's epidemic management.

\section{ACKNOWLEDGEMENT}

Our sincere and heartfelt thanks to all the authors, Bruhat Bengaluru Mahanagara Palike, Ministry of health- Government of Karnataka and Ministry of health - Government of India whose articles/reports/data are cited and included in references to this manuscript.

Conflict of interest: The authors declare that there is no conflict of interest.
Financial support: No funding.

\section{REFERENCES}

1. COVID-19 Dashboard, https://coronaboard.com/global/, accessed on 15 June 2020.

2. WHO Coronavirus Disease (COVID-19) Dashboard, https:// covid19.who.int/, accessed on 15 June 2020.

3. COVID-19 INDIA, Ministry of health and family welfare, Government of India, https://www.mohfw.gov.in/ , accessed on 15 June 2020.

4. COVID-19 Cases - Karnataka, Bruhat Bengaluru Mahanagara Palike, https://covid19.bbmpgov.in/, accessed on 15 June 2020.

5. Kannamani Ramasamy and Sudershan Reddy (2020), "The significant challenges in Bangalore - An introspection". Adalya Journal.VOLUME-9-ISSUE-4-APRIL-2020.

6. BBMP / COVID-19 WAR ROOM, https://dl.bbmpgov.in/covid/, accessed on 15 June 2020.

7. Government of Karnataka, Karnataka State COVID-19 War Room, Daily Report COVID-19 Positive Cases Analysis of Karnataka State, https://covid19.karnataka.gov.in, accessed on 15 June 2020.

8. Create a forecast in Excel for Windows, https://support.office. com/en-us/article/create-a-forecast-in-excel-for-windows22c500da-6da7-45e5-bfdc-60a7062329fd, accessed on 15 June 2020.

9. India's COVID-19 Testing Capacity Must Grow by a Factor of 10: Here's How That Can Happen, https://www.cgdev.org/ publication/indias-covid-19-testing-capacity-must-grow-factor10-heres-how-can-happen, accessed on 15 June 2020.

10. Rate of COVID-19 testing in most impacted countries worldwide as of 15 June, 2020, https://www.statista.com/statistics/1104645/covid19-testing-rate-select-countries-worldwide/ , accessed on 15 June 2020.

11. How Bengaluru kept its coronavirus count low, https://timesofindia.indiatimes.com/city/bengaluru/how-bengaluru-kept-its-coronavirus-count-low/articleshow/76240490.cms , accessed on 15 June 2020.

12. Kannamani Ramasamy, S. Jayakumar, Govindasamy Chinnu. COVID-19 Situation at Chennai City - Forecasting for the Better Pandemic Management International Journal of Current Research and Review. Vol 12 Issue 12, June 2020, 37-47, doi: http://dx.doi.org/10.31782/IJCRR.2020.12128 